\title{
Sphenoid sinus septations and their interconnections with parasphenoidal internal carotid artery protuberance: radioanatomical study with literature review
}

\author{
Tomasz Dziedzic ${ }^{1}$, Kacper Koczyk ${ }^{1}$, Tomasz Gotlib ${ }^{2}$, Przemysław Kunert ${ }^{1}$, Edyta Maj ${ }^{3}$, Andrzej Marchel $^{1}$ \\ ${ }^{1}$ Department of Neurosurgery, Medical University of Warsaw, Warsaw, Poland \\ ${ }^{2}$ Department of Otolaryngology, Medical University of Warsaw, Warsaw, Poland \\ ${ }^{3} 2^{\text {nd }}$ Department of Clinical Radiology, Medical University of Warsaw, Warsaw, Poland
}

Videosurgery Miniinv 2020; 15 (1): 227-233

DOI: https://doi.org/10.5114/wiitm.2019.85837

\begin{abstract}
Introduction: Internal carotid artery (ICA) injury is the most dangerous and life-threatening complication in patients operated on due to parasellar tumors via a minimally invasive endoscopic endonasal approach. Sphenoid septal attachment to the ICA protuberance within the sphenoid sinus was found to be one of the anatomical risk factors for ICA injury during transsphenoidal surgery.

Aim: To determine the relationship between the sphenoid sinus septa and the parasellar or paraclival internal carotid artery prominence based on our own material and a literature review.

Material and methods: The axial plane scans of computed tomography angiography and a literature review of previously published papers on the septum variation and its connection with the ICA prominence are provided.

Results: Out of 100 sphenoid sinuses, 49 (49\%) had at least one septum inserted at the ICA prominence. In the majority of cases 42 (86\%) one septum was inserted at the prominence of one of the ICAs. In 7 (14\%) cases, two separate septa were inserted at the prominences of both ICAs. Patients with multiple septa and those having an incomplete septum were at higher risk of at least one of them being inserted at the ICA prominence within the sinus. Including cases from the literature review, the average number of septa per patient was 1.42. The risk of intersection between the septum and the ICA prominence was 32\%.
\end{abstract}

Conclusions: A significant percentage of the intrasphenoidal septa are inserted at the sphenoidal ICA protuberance.

Key words: endoscopic, endonasal, skull base, sphenoid sinus, sphenoidotomy, internal carotid artery.

\section{Introduction}

Extended endoscopic endonasal approaches for skull base pathologies are gaining popularity among neurosurgeons due to a relatively low rate of complications and satisfactory results in terms of tumor resection and patients' quality of life in the postoperative period [1]. Anatomical variations of the sphenoid sinus anatomy in relation to the optic nerve and internal carotid artery (ICA) are of great importance. Internal carotid artery injury is the most dangerous and life-threatening complication in patients operated on due to parasellar tumors. Sphenoid septal attachment to the ICA protuberance within the sphenoid sinus was found to be one of the anatomical risk factors for ICA injury during transsphenoidal surgery [2]. In previous publications this anatomical relationship was found in only $11 \%$ or as many as in $87 \%$ of cases $[3,4]$. Due 
to this uncertainty in the literature, we decided to reevaluate this problem.

\section{Aim}

The aim of the study was to determine the anatomical relationship between the sphenoidal sinus septation patterns and the ICA course based on our own material and a literature review.

\section{Material and methods}

In the first part of the study, we analyzed radiological documentation of 100 patients who were treated in our department. We included only adults: 65 women and 35 men. Their age varied from 20 to 87 , with the mean age of all patients being 55.6 years. Computed tomography angiography (CTA) scans of patients with sellar or presellar types of sphenoid sinus pneumatization according to the classification presented by Hammer and Radberg were included in the study [5]. Patients with a conchal type of sphenoid sinus were excluded from the study (2 patients). In all cases CTA scans were done due to other than skull base tumor pathology. All angiograms were performed with $0.6 \mathrm{~mm}$ separation acquisition, and for the purpose of the study axial plane scans were reviewed. Each scan was assessed for sphenoid sinus septa quantity, its pattern and their relation to the ICA protuberance within the sphenoid sinus. During the assessment, complete and incomplete septa were taken into consideration. The differentiation between complete and incomplete septa was based on their simultaneous contact with the anterior and posterior sphenoid sinus wall. During the assessment of the pattern of the septa, those with one dominating main trunk and small branches were called "branched". Each type of septal pattern is presented in Photo 1. Each exam was analyzed at least three times by two independent researchers. Where two researchers had different opinions, the final decision was based on consensus.

A review of the literature using the MEDLINE database was performed to find papers on the septum variation and its connection with the ICA prominence within the sphenoid sinus. Such key phrases as "sphenoid sinus anatomy" and "sphenoid sinus septations" were used to perform the search. Inclusion criteria for review were limited to the English language. Exclusion criteria were studies which did not refer to the information about the interconnec- tion between sphenoid septa and the ICA protuberance. Any papers referenced by studies obtained from the initial Medline search were included in the study. We did not identify any review papers before this publication. Publications where it was impossible to estimate the correct number of septa were excluded from the analysis $[6,7]$. Factors such as the visualization method on which the study was based, the number of patients included in the study, the quantity of septa, average septa per patient, and the quantity of septa that had interconnection with the ICA protuberance were assessed. Since 1995, when Sethi et al. published the first paper where we found information about the interconnection between intrasphenoid septa and the ICA protuberance, we have been able to identify another 9 papers which had been published by the end of 2015. There were two purely anatomical $[8,9], 5$ radiological $[4,10-$ 13] and 3 combining those two methods of assessment $[3,4,14]$. There were studies which included patients with $[4,11,12]$ or without $[3,8-10,13,14]$ pathology within or in proximity to the sphenoid sinus. A summary of all the studies is presented in Table I.

The paper does not report on primary research. All data analyzed were collected as a part of routine diagnosis. The paper does not report on the use of experimental or new protocols. Patients were diagnosed and treated according to best medical knowledge. Due to above-mentioned reasons, ethics committee approval was not obtained.

\section{Statistical analysis}

For statistical analysis, the $\chi^{2}$ test was used to compare single septum and multiple septa groups, complete and incomplete groups and also to compare "regular septa" with "branched septa" when a normal distribution was observed. $P<0.05$ was considered statistically significant.

\section{Results}

In the 100 patients included in the study, 136 septa were identified, which gives 1,36 septa per patient. The quantity of septa within the sinus varied from none to three per patient. In $2 \%$ of sphenoid sinuses, either a complete or incomplete septum was not visualized. In the majority of cases $(63 \%)$ one septum was found. In the rest of sinuses, more than one septum was recognized (35\%). Those patients 

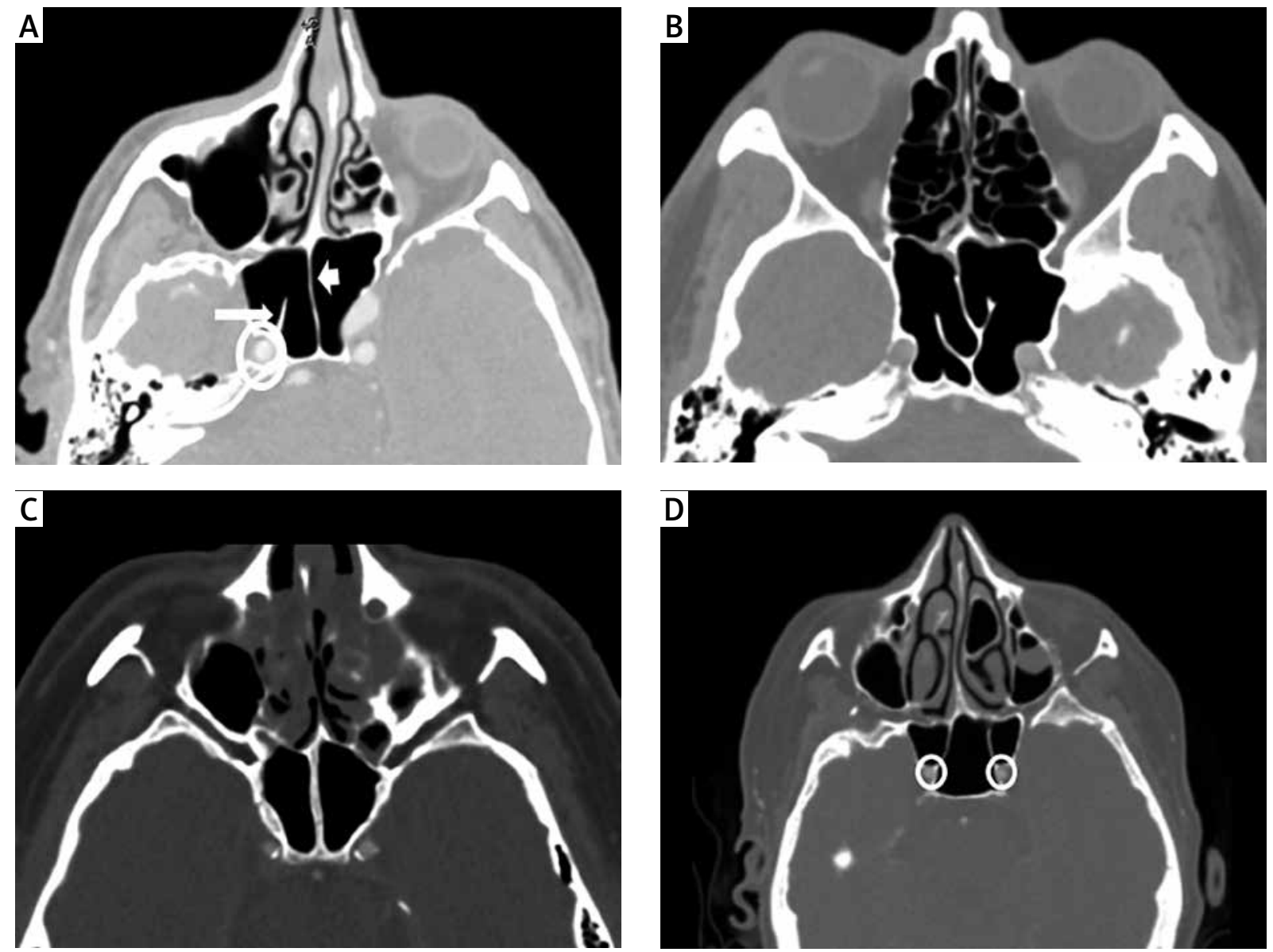

Photo 1. Computed tomography angiography representing different types of sphenoid sinus patterns. A - Short thin white arrow indicates incomplete septa; White arrow head indicates complete septa. B - Scan where "branched type" of the septum was identified. C - Scan represents "typical" midline septum which divides sinus into two comparable compartments. D - Example of the sinus with two septa that have interconnection with both ICA protuberances. On scans A and D the white circle indicates a point of interconnection between the septum and the ICA protuberance

had two $(32 \%)$ or three septa (3\%) within the sinus. One hundred out of 136 (73\%) septa were assessed as complete and 36 (27\%) as incomplete. Branched septa were identified only in patients in whom a complete septum was identified. They accounted for $15.4 \%$ of all septa and $21 \%$ out of only complete septa. In 21 (21\%) cases a typical single midline septum dividing the sphenoid sinus into two parts of comparable size was found. Out of 100 sphenoid sinuses, 49 (49\%) had at least one septum inserted at the ICA protuberance. In the majority of cases (42; $86 \%)$, one septum was inserted at the protuberance of one of the ICAs. In 7 (14\%) cases, two separate septa were inserted at both ICAs. We did not find any case where one ICA had a connection with more than one septum. Septa were more often inserted in the left ICA protuberance $(23 / 42 ; 55 \%)$ than the right one (19/42; 45\%). Among patients with a single septum, its insertion at the ICA protuberance was recorded in 22 (35\%) cases. In patients with multiple septa there was a higher rate that at least one of them would be inserted at the parasellar or paraclival ICA prominence. This pattern was found in 33 out of 35 (94\%) cases. When 3 septa were identified in all cases there was at least one interconnection between the septum and the ICA. The presence of multiple septa is statistically significant in terms of the risk of interconnections between the septum and the ICA protuberance $(p<0.05)$. The presence of an incomplete septum was also related to a higher incidence $(p<0.05)$ of connection between the septum and the ICA protuberance. This was pres- 


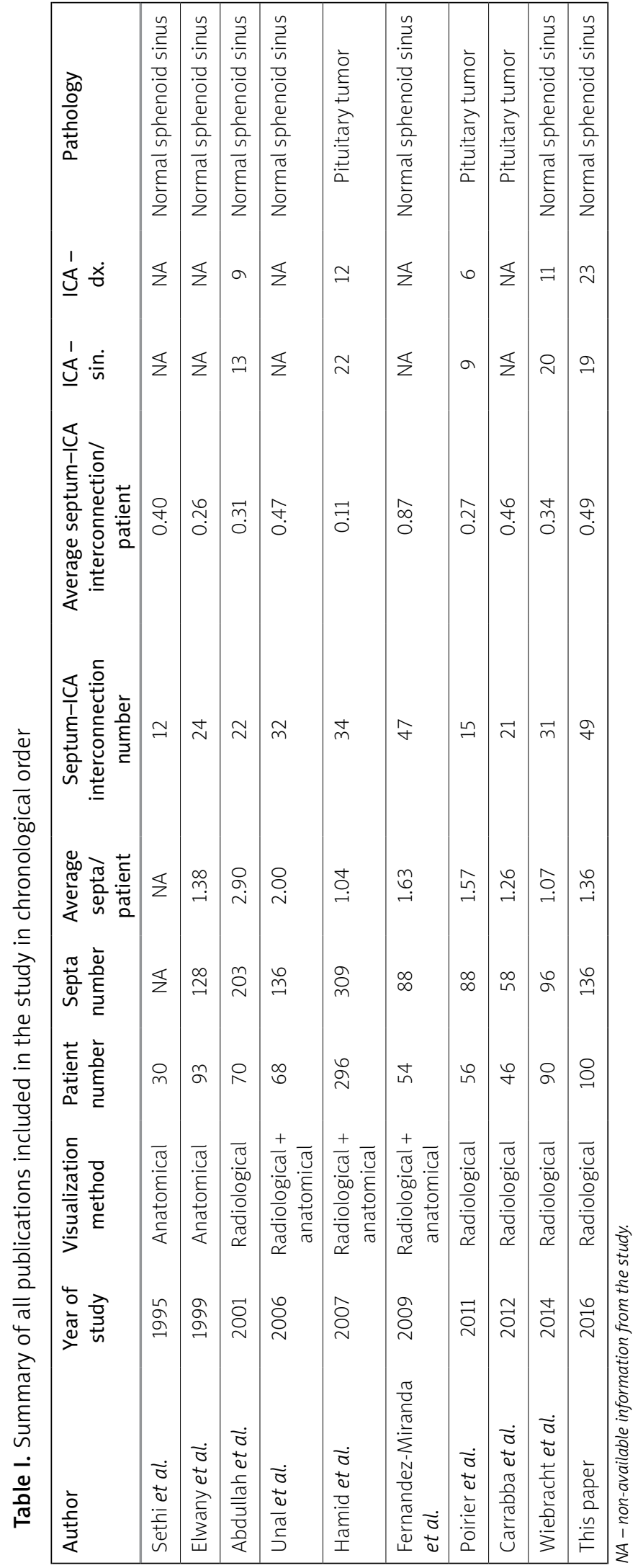

ent in 22/36 (61\%) of cases of incomplete and in $36 / 100(36 \%)$ of complete septum. A similar rate of interconnection between the septum and the ICA protuberance was found when regular septa were compared to "branched septa". An interconnection was found in 33 out of 79 (41.7\%) sphenoid septa cases with regular septation patterns, and in 9 out of $21(42.8 \%)$ where branched septa were recorded. This difference was not statistically significant $(p=$ 0.93) (Table II).

In total (including this paper) we were able to identify 903 patients (Table I). In those patients, excluding the paper of Sethi et al., we identified 1242 septa. The average number of septa per patient was 1.42. In 903 patients in 287 cases of sphenoid sinuses, we were able to identify at least one septum that had an interconnection with the ICA protuberance within the sphenoid sinus. This gives the risk of interconnection between the septum and the ICA protuberance of $32 \%$. The left and right ICA protuberance had an interconnection with septum in 58\% and $42 \%$ of cases, respectively.

\section{Discussion}

Extended endoscopic endonasal approaches usually require a wide opening of the sphenoid sinus for sufficient visualization of the posterior wall of the sinus and to provide easy access to the operated region. This step often requires the removal of the septa from the sphenoid sinus. During the sphenoid sinus opening and also when a wide opening of the sella turcica is required, special attention should be paid to the ICA anatomical course.

Our study included 100 adult Caucasian patients, both men and women. Gender and ethnic group does not seem to influence the septa quantity and septation patterns within the sphenoid sinus [10]. We included only adult patients but similar observations can be made for patients over 12 years of age, when the sphenoid sinus reaches its full size and final anatomical configuration. This is the time when the process of pneumatization, which usually starts 12 months after birth, ends [15]. In none of the presented patients was there a history of pituitary or parasellar tumor, which may affect the anatomy of the sinus. For the purpose of our study we used CTA axial scans, which were found to be superior in most studies in terms of septa identification in preoperative imaging, and completely repetitive when 
Table II. Summary of the results from the present study $(n=100)$

\begin{tabular}{|c|c|c|}
\hline \multirow[t]{4}{*}{ Septa quantity } & \multirow[t]{4}{*}{ In total - 136 septa } & 0 septum within sinus $-2(2 \%)$ \\
\hline & & 1 septum within sinus - $63(63 \%)$ \\
\hline & & 2 septa within sinus - $32(32 \%)$ \\
\hline & & 3 septa within sinus $-3(3 \%)$ \\
\hline \multirow[t]{3}{*}{ Septa pattern } & \multicolumn{2}{|l|}{ Incomplete - 36 septa } \\
\hline & \multirow[t]{2}{*}{ Complete -100 septa } & Branched septum - 21 (21\%) \\
\hline & & Regular septum - 79 (79\%) \\
\hline \multirow[t]{3}{*}{ Septum-ICA interconnection } & \multirow[t]{2}{*}{ (+) $49(49 \%)$ sinuses } & Left ICA - 19 (45\%) \\
\hline & & Right ICA - $23(55 \%)$ \\
\hline & \multicolumn{2}{|l|}{ (-) $51(51 \%)$ sinuses } \\
\hline \multirow[t]{2}{*}{$\begin{array}{l}\text { Septum quantity and the risk of } \\
\text { septum-ICA interconnection }\end{array}$} & $\begin{array}{l}\text { Single septum } \\
22 / 63(35 \%)\end{array}$ & $\begin{array}{l}\text { Multiple septa } \\
33 / 35(94 \%)\end{array}$ \\
\hline & \multicolumn{2}{|c|}{ Statistically significant difference $p<0.05$} \\
\hline \multirow[t]{2}{*}{$\begin{array}{l}\text { Septum completeness and the risk } \\
\text { of septum-ICA interconnection }\end{array}$} & $\begin{array}{l}\text { Complete septum } \\
36 / 100(36 \%)\end{array}$ & $\begin{array}{l}\text { Incomplete septum } \\
22 / 36(61 \%)\end{array}$ \\
\hline & \multicolumn{2}{|c|}{ Statistically significant difference $p<0.05$} \\
\hline \multirow[t]{2}{*}{$\begin{array}{l}\text { Septum branching and the risk of } \\
\text { septum-ICA interconnection }\end{array}$} & $\begin{array}{l}\text { Regular septum } \\
33 / 79(42 \%)\end{array}$ & $\begin{array}{l}\text { "Branched septum" } \\
\text { 9/21 (43\%) }\end{array}$ \\
\hline & \multicolumn{2}{|c|}{ Difference was not statistically significant, $p=0.93$} \\
\hline
\end{tabular}

compared with coronal scans [3, 14], although one publication found the superiority of coronal over axial scans in terms of septa assessment [10]. For proper visualization of soft tissue, magnetic resonance seems to be superior to computed tomography (CT) scans but there is no doubt that for skull base orientation CT is beneficial [14]. One of the papers was based only on MRI scans and it had to be excluded from the analysis because it was impossible to estimate the quantity of septa that had an interconnection with the ICA protuberance [7]. The computed tomography with thicker separation acquisition scans that we used in our study had been previously validated and found to be highly reproductive in terms of sphenoid sinus septation patterns when an endoscopic transsphenoidal approach was used [3]. Patients with a conchal or nonpneumatized type of pneumatization assessed on sagittal scans were excluded from the study due to lack of septations in this type of the sinus in general. Those two types of pneumatization account for about $2 \%$ of all sinuses in the literature and also in our group $[4,13,16]$.
Septa are long and thin layers that divide the sinus into compartments. They usually originate at the site of fusion of the several synchondroses of the sphenoid bone. There is a great variety of septa quantity, morphological type, their orientation within the sinus and position with respect to the midline [7]. In the present paper, the mean quantity of septa within the sinus was 1.36 septa per patient. In the literature this quantity varied from 1.04 up to as many as 2.9 per patient $[4,10]$. In $2 \%$ of sphenoid sinuses, neither a complete nor an incomplete septum was visualized. In a similar percentage of patients in the available literature, a lack of septa within the well-pneumatized sinus has been recognized [10]. However, in one study no septa within the sinus was recognized in almost $11 \%$ of patients [4]. In most of the papers as well as in the present paper, most frequently only one septum is recognized [4]. From the reviewed papers, the highest reported number of septa within the sphenoid sinus was 6 [10]. Sphenoid sinus septa quantity seems to be higher in patients with acromegaly and there is also a high- 
er tendency for the conchal type of pneumatization in those patients and those septa inserts at the ICA protuberance [11].

The typical midline septum, which divides the sinus into two comparable compartments was seen in $21 \%$ of cases in the present group, which is similar to the paper by Zada et al. and significantly more common than in the paper presented by FernandezMiranda et al. [3, 7]. Contrary to the typical midline textbook definition of sphenoid sinus septations, most septa are asymmetrical [17]. According to those findings, other anatomical landmarks should be used as a midline landmark, which is of great importance during a transsphenoidal procedure. According to the present and the previous literature, the vomer seems to be the best landmark for the midline orientation [7]. Other structures, such as the midline filum of the sellar dura, can also be useful according to Zada et al. [18].

The percentage of sphenoid sinuses where at least one septum was involved with the ICA protuberance was $49 \%$ in the present paper. In the available literature, the percentage of this interconnection ranges from $11 \%$ to $87 \%[3,4,10]$. In our paper and in those we reviewed, usually only one ICA protuberance had an interconnection with the septum. In the paper of Poirier et al. both ICAs were involved in 3.4\% of the cases, which is a lower rate when compared with the our material, 14\% [12]. In none of our cases, and in those we reviewed only one ICA had a connection with more than one septum. Patients with multiple septa have a higher risk that one of the septa will connect with the ICA protuberance. The risk of the septum having an interconnection was related to the number of the septa within the sinus. In the case of 3 septa, at least one of them was found to have this kind of interconnection. In the paper of Abdullah et al. patients with multiple septa accounted for $81.8 \%$ of sinuses with an interconnection between the septa and the ICA protuberance, and in the paper of Hamid et al. the presence of accessory septa was also found to be more common when in connection with the ICA $[4,10]$. The second factor that may point to an increased risk is presence of incomplete septa. Incomplete septa in some papers was defined as an accessory septa. In the paper of Fernandez-Miranda et al. those two types of septation patterns were identified but there was no information about the incidence of interconnection between the ICA protuberance and septa [3]. In patients where a "branched" septum was visualized, we did not find that this type of septum was more highly predisposed to have an interconnection with the ICA protuberance. This type of septum in its description is similar to the term "complex" septum in the papers that we reviewed.

During surgery the surgeon has to be familiar with the patient's sphenoidal sinus septal anatomy before any attempts to remove it are made. As mentioned before, it may be attached to the ICA protuberance or to the optic nerve. Septum drilling instead of septum fracture should be a preferred method for its removal $[3,10]$. Several authors pointed out that this maneuver is very risky in cases where the septum has a connection with the ICA prominence $[19,20]$. The removal of the septa should be ideally performed using a high speed drill and in a parallel direction to the course of the ICA using a 3 or $4 \mathrm{~mm}$ diamond burr [3]. One should keep in mind that complete septal removal is not always required for proper sella visualization due to the fact that their origin is usually lateral to the sella turcica [21]. Another important fact about the surgery around the sphenoid sinus and ICAs is the proximity of the ICA to the midline. In some cases it can be as close as $4 \mathrm{~mm}$ [17]. In those cases the transcranial route should considered. Patients with acromegaly are at increased risk of this unfavorable anatomical configuration [11]. Additionally, attention should be paid to the thin bony wall over the ICA within the sinus or its dehiscence over the ICA and when it is covered only by sphenoidal mucosa. This is seen in up to $25 \%$ of cases based on radiological and in almost $5 \%$ of cases in anatomical studies $[8,10]$. In our unpublished material, lack of bony covering around the ICA within the sphenoid sinus was found in $9 \%$ of cases based on radiological study. In all cases this dehiscence is found in the pre-sellar segment, which is adjacent to the anterior wall of the sella [8].

\section{Conclusions}

Each presented paper, including this one, is based on a relatively small number of patients and each was based on studies on patients from different ethnic groups. In our opinion, this analysis of almost one thousand patients gives more complex information about this topic by combining different techniques and different ethnic groups. We can say that the average number of septa within the sinus is more than one. The risk of at least one septum hav- 
ing an interconnection with the ICA protuberance is estimated to be $32 \%$.

\section{Conflict of interest}

The authors declare no conflict of interest.

\section{References}

1. Little AS, Kelly D, Milligan J, et al. Predictors of sinonasal quality of life and nasal morbidity after fully endoscopic transsphenoidal surgery. J Neurosurg 2015; 122: 1458-65.

2. Valentine R, Wormald PJ. Carotid artery injury after endonasal surgery. Otolaryngol Clin North Am 2011; 44: 1059-79.

3. Fernandez-Miranda JC, Prevedello DM, Madhok R, et al. Sphenoid septations and their relationship with internal carotid arteries: anatomical and radiological study. Laryngoscope 2009; 119: 1893-6.

4. Hamid O, El Fiky L, Hassan O, et al. Anatomic variations of the sphenoid sinus and their impact on trans-sphenoid pituitary surgery. Skull Base 2008; 18: 9-15.

5. Hammer G, Radberg C. The sphenoidal sinus. An anatomical and roentgenologic study with reference to transsphenoid hypophysectomy. Acta Radiol 1961; 56: 401-22.

6. Chougule MS, Dixit D. A cross-sectional study of sphenoid sinus through gross and endoscopic dissection in North Karnataka, India. J Clin Diagn Res 2014; 8: Ac01-05.

7. Zada G, Agarwalla PK, Mukundan S Jr, et al. The neurosurgical anatomy of the sphenoid sinus and sellar floor in endoscopic transsphenoidal surgery. J Neurosurg 2011; 114: 1319-30.

8. Elwany S, Elsaeid I, Thabet H. Endoscopic anatomy of the sphenoid sinus. J Laryngol Otol 1999; 113: 122-6.

9. Sethi DS, Stanley RE, Pillay PK. Endoscopic anatomy of the sphenoid sinus and sella turcica. J Laryngol Otol 1995; 109: 951-5.

10. Abdullah B, Arasaratnam S, Kumar G, Gopala K. The sphenoid sinuses: computed tomographic assessment of septation, relationship to the internal carotid arteries, and sidewall thickness in the Malaysian population. J HK Coll Radiol 2001; 4: 185-8.

11. Carrabba G, Locatelli M, Mattei L, et al. Transphenoidal surgery in acromegalic patients: anatomical considerations and potential pitfalls. Acta Neurochir (Wien) 2013; 155: 125-30.

12. Poirier J, Duggal N, Lee D, Rotenberg B. Sphenoid sinus septations: unpredictable anatomic landmarks in endoscopic pituitary surgery. J Otolaryngol Head Neck Surg 2011; 40: 489-92.

13. Wiebracht ND, Zimmer LA. Complex anatomy of the sphenoid sinus: a radiographic study and literature review. J Neurol Surg B Skull Base 2014; 75: 378-82.

14. Unal B, Bademci G, Bilgili YK, et al. Risky anatomic variations of sphenoid sinus for surgery. Surg Radiol Anat 2006; 28: 195-201.

15. Reittner P, Doerfler O, Goritschnig T, et al. Magnetic resonance imaging patterns of the development of the sphenoid sinus: a review of 800 patients. Rhinology 2001; 39: 121-4.

16. Vaezi A, Cardenas E, Pinheiro-Neto C, et al. Classification of sphenoid sinus pneumatization: relevance for endoscopic skull base surgery. Laryngoscope 2015; 125: 577-81.

17. Renn WH, Rhoton AL Jr. Microsurgical anatomy of the sellar region. J Neurosurg 1975; 43: 288-98.
18. Zada G, Kim AH, Governale LS, Laws ER. Midline filum of the sellar dura: a useful landmark during endoscopic transsphenoidal pituitary surgery. Neurosurgery 2010; 67: 391-4.

19. Laws ER Jr. Vascular complications of transsphenoidal surgery. Pituitary 1999; 2: 163-70.

20. Kassam A, Snyderman CH, Mintz A, et al. Expanded endonasal approach: the rostrocaudal axis. Part I. Crista galli to the sella turcica. Neurosurg Focus 2005; 19: E3.

21. Shah NJ, Navnit M, Deopujari CE, Mukerji SS. Endoscopic pituitary surgery - a beginner's guide. Indian J Otolaryngol Head Neck Surg 2004; 56: 71-8.

Received: 25.04.2019, accepted: 12.05.2019. 